\title{
NaCl溶液浓度及其腐蚀作用对II型水合物颗粒 黏附力影响
}

\author{
王盛龙 ${ }^{1}$, 㚞栓狮 ${ }^{*}$, 宋永臣 ${ }^{2}$, 郎雪梅 ${ }^{1}$, 王燕鸿 ${ }^{1}$, 李刚 ${ }^{1}$, 陈建标 ${ }^{1}$ \\ 1. 华南理工大学化学与化工学院, 传热强化与过程节能教育部重点实验室, 广州 510640; \\ 2. 大连理工大学能源与动力学院, 海洋能源利用与节能教育部重点实验室, 大连 116024 \\ *联系人, E-mail: ssfan@scut.edu.cn
}

收稿日期: 2018-05-02; 接受日期: 2018-06-26; 网络出版日期: 2018-10-08

国家自然科学基金重点项目(编号: 21736005)和国家重点研发计划(编号: 2017YFC0307303-2, 2017YFC0307302-2, 2016YFC0304006)资助

\begin{abstract}
摘要 在天然气水合物资源开采和深水油气输运过程中, 管道内的低温高压环境会导致气体水合物颗粒的生成 和聚集并最终造成管道堵塞，严重影响生产效率。导致这一问题的根本原因是气体水合物颗粒间以及颗粒-表面 间存在黏附力. 因此, 为了探明气体水合物在管道中的沉积和聚集机理, 本文利用高压微机械力测量装置, 对 $\mathrm{CH}_{4} / \mathrm{C}_{2} \mathrm{H}_{6}$ 混合气生成的II型水合物颗粒在不同盐度和碳钢表面腐蚀程度影响下的黏附力进行了原位测量. 实验 结果表明 $\mathrm{NaCl}(1 \mathrm{wt} . \%-5 \mathrm{wt}$ \% \%溶液)的存在能够降低水合物颗粒间的黏附力，根据毛细液桥理论模型估算结果， 出现这一现象的可能原因是 $\mathrm{NaCl}$ 的存在降低了液桥与水合物颗粒表面的接触角。此外，经 $\mathrm{NaCl}$ 溶液腐蚀处理后， 碳钢表面与水合物颗粒间的黏附力显著上升, 最多可升高至无腐蚀条件下颗粒-碳钢表面间黏附力的 5 倍左右.
\end{abstract}

关键词 天然气水合物, 黏附力, 盐度, 腐蚀, 流动安全

PACS: 89.30.an, 89.30.aj, 61.46.Df, 81.70.Fy, 51.10.+y

\section{1 引言}

天然气水合物通常是指由天然气中的主要成分 $\left(\mathrm{CH}_{4}, \mathrm{C}_{2} \mathrm{H}_{6}\right.$ 等气体)与水在高压低温条件下形成的非化 学计量笼形络合物 ${ }^{[1]}$. 在水合物资源开采和油气输运 过程中，管道中流体的组分包含天然气和水。在这种 流动状态下，由于管道中的低温高压条件，液相中微 小的气泡以及气相中凝结的水滴可能形成水合物颗 粒. 水合物颗粒在随流体流动过程中会发生聚集并可
能附着于管壁，最终堵塞管道，造成局部压力升高、 开采效率降低并导致潜在的安全问题. 典型的管道内 水合物堵塞形成过程由以下4个阶段构成(如图1所 示 $)^{[2]}:(1)$ 气泡在液相中的悬浮以及液滴在管道壁面的 凝结; (2) 水合物颗粒在液相和壁面的成核及生长; (3) 水合物颗粒在流动中的聚集与沉积; (4) 沉积物体积逐 渐增加, 最终堵塞管道 ${ }^{[2]}$. 在上述过程中, 导致水合物 发生聚集和沉积的根本原因是水合物颗粒间以及水合 物颗粒与管道壁面间的黏附力 ${ }^{[3-5]}$.

引用格式: 王盛龙, 㚞栓狮, 宋永臣, 等. $\mathrm{NaCl}$ 溶液浓度及其腐蚀作用对II型水合物颗粒黏附力影响. 中国科学: 物理学 力学 天文学, 2019, 49: 034608 Wang S L, Fan S S, Song Y C, et al. The effect of $\mathrm{NaCl}$ concentration and corrosion on cohesion/adhesion forces of structure-II hydrate particles (in Chinese). Sci Sin-Phys Mech Astron, 2019, 49: 034608, doi: 10.1360/SSPMA2018-00169 


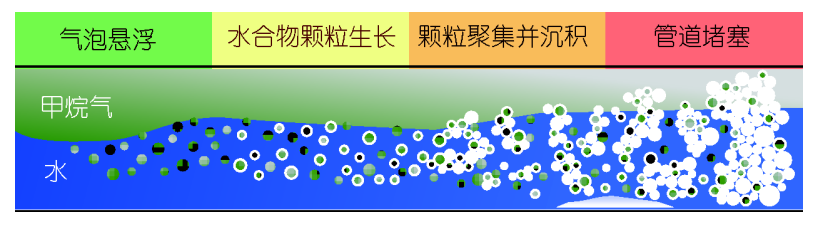

图 1 (网络版彩图)管道内水合物堵塞形成的典型过程(根 据Turner等人 ${ }^{[6]}$ 改编)

Figure 1 (Color online) Conceptual picture of hydrate plug formation in flowlines [6].

因此，为了研究水合物颗粒间以及水合物颗粒与 管壁间的微观作用机理并探究其影响因素, 研究人员 针对水合物颗粒-颗粒间以及颗粒-表面间黏附力展开 了大量研究. 由于环戊烷 $\left(\mathrm{CyC}_{5}\right)$ 及四氢呋喃(THF)这一 类物质常压下即可生成水合物，具有容易操作和观测 的优点，研究者们首先对这一类水合物颗粒间以及颗 粒-表面间的相互作用进行了研究. Yang等人 ${ }^{[7]}$ 研究了 温度对于冰颗粒和 THF水合物颗粒间作用力的影响, 发现在 $-10^{\circ} \mathrm{C}-2^{\circ} \mathrm{C}$ 之间, THF水合物颗粒间作用力随 着温度的降低而降低, 并且冰颗粒与 THF颗粒的作用 力在相同条件下具有近似的变化规律. Taylor等人 ${ }^{[8]}$ 在 Yang等人 ${ }^{[7]}$ 的研究基础上测量了正癸烷中THF水合物 颗粒间的作用力，发现水合物颗粒间作用力不仅与温 度有关，还与颗粒间的接触力、接触时间以及所处介 质的界面能成比例. Dieker等人 ${ }^{[9]}$ 测量了原油存在条 件下 $3.2^{\circ} \mathrm{C}$ 时 $\mathrm{CyC}_{5}$ 水合物颗粒间的相互作用力, 发现 较纯水体系中的 $\mathrm{CyC}_{5}$ 水合物颗粒间作用力有所降低, 其数值在 $0.5 \mathrm{mN} / \mathrm{m}$ 左右. Brown等人 ${ }^{[10]}$ 测试了 $\mathrm{CyC}_{5}$ 水 合物生成后的保持时间对于水合物颗粒间作用力的影 响，发现颗粒间作用力在不同温度下均出现了随保持 时间延长而降低的现象. Lee等人 ${ }^{[11]}$ 研究了热力学抑 制剂存在条件下 $\mathrm{CyC}_{5}$ 水合物颗粒间的作用力, 发现不 同热力学抑制剂虽然可以改变水合物相平衡条件, 使 水合物生成过程需要更大的驱动力，但同时也会增加 水合物颗粒间的作用力.

当水合物颗粒聚集的体积逐渐增大时，聚集体会 在管道中发生沉积, 并附着在管壁上. 此外, 管壁的冷 凝液滴等在低温高压条件下也会自发转化为水合物颗 粒. 因此, 为了探明水合物颗粒和不同固体表面间的微 观作用机理，Aspenes 等人 ${ }^{[12]}$ 测量了 $\mathrm{CyC}_{5}$ 水合物颗粒 与不锈钢、黄铜和铝等固体表面间的附着力, 其结果 表明界面能越低的固体表面与水合物颗粒间的附着力 越小, 而当水合物颗粒与固体表面间存在自由水时, 两
者之间的作用力显著增大. Smith等人 ${ }^{[13]}$ 在进行 THF水 合物颗粒与钢表面间作用力实验时也得到了类似结 果，并基于此提出了一种通过降低表面能从而减小水 合物颗粒与固体表面间作用力的表面处理方法. Aman 等人 ${ }^{[14,15]}$ 测量了 $\mathrm{CyC}_{5}$ 水合物颗粒间的作用力及颗粒石英表面间的作用力, 并采用了与Smith等人 ${ }^{[13]}$ 类似的 表面处理方法以降低两者之间的作用力.

尽管针对常压水合物颗粒作用力的研究已经比较 完善, 但实际管道内气-水流动过程中生成的多为气体 水合物颗粒, 上述研究以 $\mathrm{CyC}_{5} / \mathrm{THF}$ 等常压水合物为研 究对象, 其结果难以直接用于分析实际管路中气体水 合物颗粒的聚集和沉积过程. 仅有少量研究者关注了 气体水合物颗粒间的作用力并进行了初步研究. Lee 等人 ${ }^{[16]}$ 对 $\mathrm{CH}_{4}$ 和 $\mathrm{CO}_{2}$ 水合物颗粒间作用力进行了测量, 发现在保持时间 $>2.5 \mathrm{~h}$ 条件下, $\mathrm{CO}_{2}$ 水合物颗粒间作用 力为 $30 \mathrm{mN} / \mathrm{m}$ 左右, 和 $\mathrm{CH}_{4}$ 水合物颗粒间作用力相当 $(33 \mathrm{mN} / \mathrm{m}$ 左右). 此外, 在海洋水合物资源开采和海底 油气输运过程中, 深水管道内的流体包含大量海水, 流 体盐度变化可能影响管壁水合物薄层生长、管壁腐蚀 过程和水合物颗粒的聚集过程, 因此, 分析自由水、表 面腐蚀和盐度等因素对于探明气体水合物颗粒间以及 颗粒和管道表面的黏附力影响机制及含天然气深水管 道中水合物颗粒聚集和沉积机理具有重要的推动作 用. 本文在已有研究基础上, 针对上述问题展开研究, 测量并分析了 $\mathrm{CH}_{4} / \mathrm{C}_{2} \mathrm{H}_{6}$ 水合物颗粒间以及颗粒-碳钢 表面间的黏附力, 讨论了液滴盐度和碳钢表面腐蚀对 这一黏附力的影响机制, 填补了高压条件下气体水合 物颗粒黏附力变化规律的研究空白.

\section{2 实验部分}

\section{1 实验系统与材料}

本文利用高压微机械力测量系统实现高压条件下 气体水合物颗粒间以及颗粒-表面间黏附力的直接测 量. 系统各主要部分连接关系如图2所示. 该系统由主 压力釜、气体注入与排出管路、控温系统和数据采集 系统几部分组成. 其中主压力釜的作用是为气体水合 物颗粒提供可以稳定存在的低温高压环境, 其最高工 作压力为 $10 \mathrm{MPa}$, 最低工作温度为 $-10^{\circ} \mathrm{C}$.

主压力釜中装有一个微米级可移动操纵杆和一个 固定在上端盖上的悬臂. 当进行水合物颗粒间黏附力 


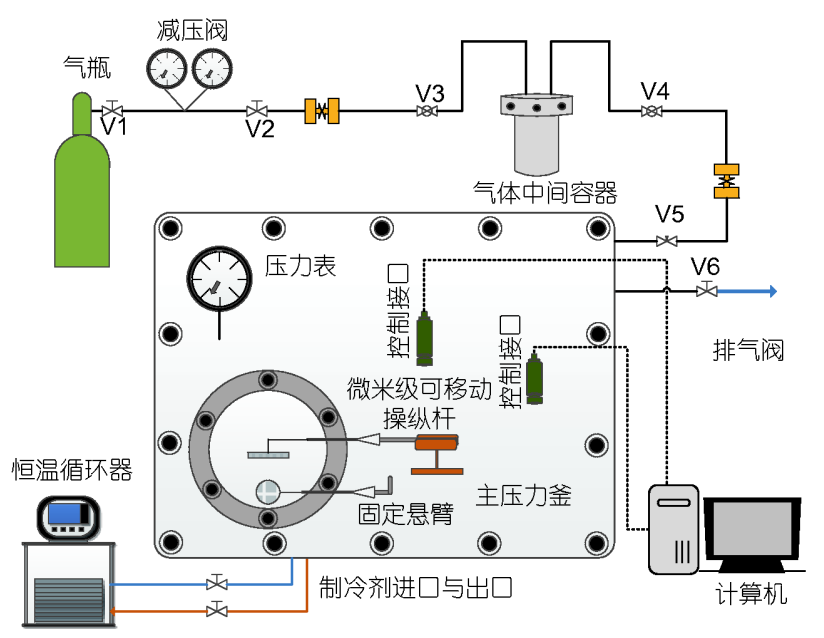

图 2 (网络版彩图)高压微机械力测量系统示意图

Figure 2 (Color online) Experimental setup of high-pressure micromechanical force apparatus.

测量时，上述两个装置端部的玻璃纤维上将固定两个 水合物颗粒，当进行水合物颗粒与界面间的黏附力测 量时，可移动操纵杆端部的水合物颗粒将更换为待测 表面样品，本文中该样品为经过不同程度表面腐蚀处 理的碳钢片. 实验过程中利用显微镜及安装在显微镜 上的高速 $\mathrm{CCD}$ 传感器透过压力釜上端盖的视窗对实 验过程进行观测和记录. 主压力釜的外壁为双层结构, 冷却液可在夹层空间中流动以保证实验过程中的温度 稳定. 实验中使用的主要材料如表1所示.

\section{2 气体水合物颗粒间及颗粒-表面间黏附力测量 方法}

\subsection{1 气体水合物颗粒生成}

为了探明液滴盐度对气体水合物颗粒间黏附力的 影响机制，实验中采用 4 种不同浓度的 $\mathrm{NaCl}$ 溶液液滴 生成水合物颗粒. 由于 $\mathrm{NaCl}$ 的加入会降低相同压力下 水合物的相平衡温度，因此，实验中控制水合物生成 过冷度相同, 以确保不同浓度 $\mathrm{NaCl}$ 溶液液滴生成的水

表 1 实验使用的主要材料

Table 1 Experimental materials

\begin{tabular}{cccc}
\hline 名称 & 组分/型号 & 纯度 $(\%)$ & 生产厂商 \\
\hline $\mathrm{CH}_{4}-\mathrm{C}_{2} \mathrm{H}_{6}$ 混合 & $74.3 \% \mathrm{CH}_{4^{-}}$ & 99.99 & General Air \\
氯化钠 & $25.7 \% \mathrm{C}_{2} \mathrm{H}_{6}$ & & \\
碳钢片样品 & $\mathrm{NaCl}$ & 99.3 & Fisher Scientific \\
\hline
\end{tabular}

合物颗粒具有相近的驱动力和转化程度, 具体参数如 表 2 所示. 气体水合物颗粒生成具体方法如下: 首先在 操纵杆或固定悬臂端部的玻璃纤维上滴一 $\mathrm{NaCl}$ 溶液 液滴, 随后, 将液滴放入主压力釜并密封, 将主压力釜 加压降温至预设的实验温度和压力后，等待液滴转化 为水合物颗粒.

\subsection{2 水合物颗粒间黏附力测量与计算}

水合物颗粒间黏附力测量步骤如图3所示. 在水合 物颗粒生成后, 设定纳米可移动操作杆的进动步长, 选 取合适的移动速度并将固定在操纵杆端部的水合物颗 粒(下称“可动颗粒”)匀速移向固定在静止悬臂(上端 盖)上的水合物颗粒(下称“固定颗粒”)(步骤1); 当两个 水合物颗粒发生接触后继续移动操纵杆一段距离, 向 固定颗粒施加预压力, 由此造成的玻璃纤维形变为 $\Delta \operatorname{Pr}$ (步骤2); 保持上述接触状态 $10 \mathrm{~s}$ 后, 向反方向移动 操纵杆, 此时由于水合物颗粒间黏附力的存在固定颗 粒将随着可动颗粒一起移动(步骤 3 ); 移动操纵杆直至 固定颗粒与可动颗粒分离, 在分离瞬间两颗粒接触点 与步骤 1 中的初始接触点间的距离记为 $\Delta D s$ (步骤4).

表 $2 \mathrm{NaCl}$ 存在条件下水合物颗粒间作用力测量实验参数 ${ }^{\mathrm{a})}$ Table 2 Experimental conditions for hydrate with $\mathrm{NaCl}^{\text {a) }}$

\begin{tabular}{cccccc}
\hline $\mathrm{NaCl}$ 浓度 & $\begin{array}{c}\text { 压力 } \\
(\mathrm{MPa})\end{array}$ & $\begin{array}{c}\text { 相平衡 } \\
\text { 温度 }\left({ }^{\circ} \mathrm{C}\right)\end{array}$ & $\begin{array}{c}\text { 实验温度 } \\
\left({ }^{\circ} \mathrm{C}\right)\end{array}$ & $\begin{array}{c}\text { 过冷度 } \\
\left({ }^{\circ} \mathrm{C}\right)\end{array}$ & $\begin{array}{c}\text { 水合物相中 } \\
\mathrm{CH}_{4} / \mathrm{C}_{2} \mathrm{H}_{6} \text { 的 } \\
\text { 摩尔比 }\end{array}$ \\
\hline 0 wt.\% & 2.1 & 6.8 & 1.4 & 5.4 & 1.48 \\
1.0 wt.\% & 2.1 & 6.4 & 1.0 & 5.4 & 1.48 \\
3.5 wt.\% & 2.1 & 5.3 & -0.1 & 5.4 & 1.49 \\
5.0 wt.\% & 2.1 & 4.7 & -0.7 & 5.4 & 1.50 \\
\hline
\end{tabular}

a) *通过CSMGem计算 ${ }^{[1]}$
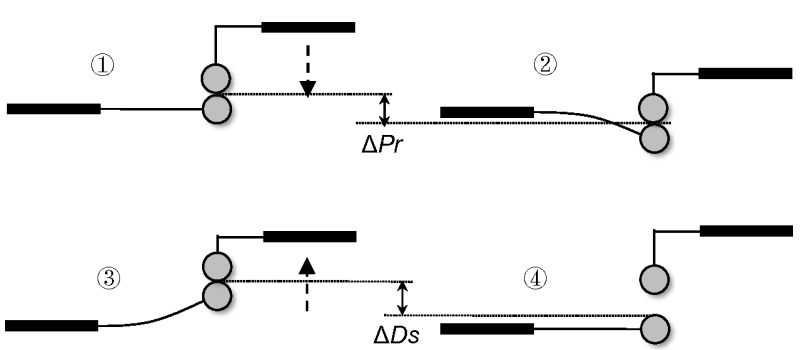

图 3 气体水合物颗粒间秥附力测量步骤

Figure 3 Procedures of gas hydrate particle cohesion force measurement. 
完成上述步骤 1 至步骤 4 记为一次测量, 且相隔两 次测量间等待 $10 \mathrm{~s}$. 颗粒间黏附力 $F$ 的计算依据胡克 定律:

$F=k \Delta D s$,

式中, $k$ 为玻璃纤维的弹性系数, 该值由预先进行的校 准实验得出. $\Delta D s$ 为水合物颗粒在与被测颗粒/表面分 离前的最大位移. 在实验过程中, 每一个特定工况下 的数据点均由 40 次以上的测量结果取平均值给出, 并 计算置信度为 $95 \%$ 的置信区间记为误差限. 考虑到多 次实验中水合物颗粒的直径各不相同，而颗粒直径的 变化会影响作用力的大小, 因此为了比较不同粒径水 合物颗粒间的黏附力, 消除颗粒尺寸对黏附力的影响, 用标准化的黏附力 $F_{\mathrm{a}}$ 代替 $F$ 作为最终的颗粒间黏附力 测量结果. 两个水合物颗粒间标准化后的作用力 $F$ 等 于测得实际作用力 $F$ 除以有效半径 $R^{*}[17]$.

$F_{\mathrm{a}}=\frac{F}{R^{*}}=\frac{F\left(R_{1}+R_{2}\right)}{2 R_{1} R_{2}}$.

\subsection{3 水合物颗粒-碳钢表面间黍附力测量与计算}

将2.2.2节颗粒间黏附力测量过程中的可动颗粒替 换为不同材质的表面，即可实现水合物颗粒与表面间 的黏附力测量. 本文为了研究碳钢表面腐蚀存在时对 水合物颗粒黏附力的影响，将可动颗粒替换为经 5 wt.\% NaCl浸泡处理的碳钢片. 在进行水合物颗粒碳钢表面间黏附力测量实验之前，需要控制测量过程 中碳钢表面的自由水量，并在碳钢片样品表面生成均 匀的水膜. 本实验中采用冷凝法, 将碳钢片表面自由 水量控制在 $90 \mu \mathrm{g} / \mathrm{mm}^{2}$ 左右(由干燥碳钢片和冷凝水后 的碳钢片分别称重获得). 随后，为了研究表面腐蚀对 颗粒-碳钢表面间的黏附力影响，碳钢片被置于 $5 \mathrm{wt} . \%$ $\mathrm{NaCl}$ 溶液中. 浸泡时长从 $0 \mathrm{~h}$ 至 $120 \mathrm{~h}$, 每间隔 $24 \mathrm{~h}$ 取出 样品一片, 从而获得6片不同表面腐蚀程度的碳钢片样 品. 最后, 对经上述方法处理过后的碳钢片与水合物颗 粒间的黏附力进行测量, 测量步骤和黏附力计算方法 与2.2.2节中所述相同.

\section{3 结果与讨论}

\section{1 液滴盐度对黏附力的影响}

$\mathrm{NaCl}$ 作为一种已知的水合物热力学抑制剂, 其加
入可以使 $\mathrm{CH}_{4} / \mathrm{C}_{2} \mathrm{H}_{6}$ 水合物的相平衡曲线向高压低温方 向移动. 然而, 目前关于 $\mathrm{NaCl}$ 的存在对水合物颗粒聚 集和沉积过程影响机制的相关研究尚处于起步阶段. 因此, 本文对不同浓度 $\mathrm{NaCl}$ 溶液生成的气体水合物颗 粒间黏附力进行了原位测量.

实验中由不同 $\mathrm{NaCl}$ 溶液生成的水合物颗粒如图4 所示. 图中可见各组水合物颗粒表面均存在不同程度 的塌陷. Servio等人 ${ }^{[18]}$ 在研究 $\mathrm{CH}_{4}$ 和 $\mathrm{CO}_{2}$ 水合物颗粒的 形态时也观察到了类似的现象. 出现这一现象的可能 原因是在水合物颗粒外壳生成完毕后, 颗粒内部的自 由水并没有完全转化为水合物, 当内部的自由水进一 步转化时, 水合物颗粒表面的局部便出现塌陷, 这也 从另一方面证明了完全生成后的水合物壳层依然可以 透过气体分子.

尽管水合物颗粒表面存在随机产生的凹陷，但对 于实验过程中发生接触的颗粒局部表面，不同浓度的 $\mathrm{NaCl}$ 液滴生成的水合物颗粒并没有明显的表面形态 区别, 光滑程度也较为一致. 在实验所用的显微镜观察 尺度内, 没有发现水合物表面粗糙程度发生明显变化, 也没有观察到树状突起(树突通常在水合物颗粒中加 入抗凝聚剂或热力学抑制剂时出现, 在 $\mathrm{CyC}_{5}$ 水合物颗 粒间作用力的研究中较为常见 ${ }^{[11]}$ ). 水合物颗粒表面出 现局部塌陷的部位不会被选择为作用力的测量部位, 因此这一随机塌陷现象对最终的实验结果并不会产生 影响.

本文首先测量了不含 $\mathrm{NaCl}$ 条件下, 即去离子水液 滴生成的 $\mathrm{CH}_{4} / \mathrm{C}_{2} \mathrm{H}_{6}$ 水合物颗粒间的黏附力, 共进行了 8 组独立重复实验, 测得的颗粒间平均作用力大小为 $(34.7 \pm 2.1) \mathrm{mN} / \mathrm{m}$, 这一数值也将作为后续实验的基准 值. 在完成基准实验后, 将生成水合物的液滴盐度由 0 wt.\%提高至 1.0 wt. \%, 进行了 5 组独立重复实验, 每 组实验包括至少 40 次的“接触-分离”测量过程. 测得的

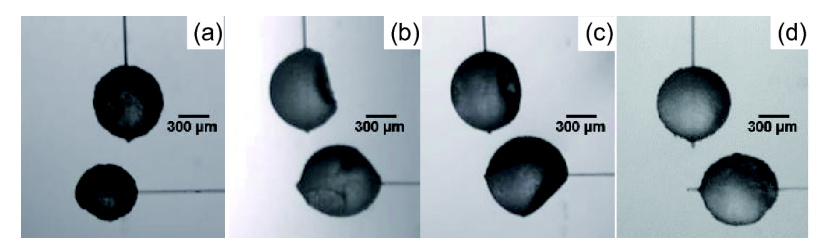

图 4 不同浓度 $\mathrm{NaCl}$ 溶液液滴生成的 $\mathrm{CH}_{4} / \mathrm{C}_{2} \mathrm{H}_{6}$ 水合物颗粒. (a) 0 wt.\%; (b) 1.0 wt.\%; (c) 3.5 wt.\%; (d) 5.0 wt.\% Figure 4 Morphology comparisons of $\mathrm{CH}_{4} / \mathrm{C}_{2} \mathrm{H}_{6}$ hydrate particles with different $\mathrm{NaCl}$ concentrations. (a) 0 wt.\%; (b) 1.0 wt.\%; (c) 3.5 wt. $\%$; (d) 5.0 wt. $\%$. 
水合物颗粒间平均作用力大小为 $(24.8 \pm 1.9) \mathrm{mN} / \mathrm{m}$ ，低 于不添加 $\mathrm{NaCl}$ 时的基准值. 这一现象表明 $\mathrm{NaCl}$ 的存在 可能导致 $\mathrm{CH}_{4} / \mathrm{C}_{2} \mathrm{H}_{6}$ 水合物颗粒间的黏附力降低.

为了进一步探究 $\mathrm{NaCl}$ 的加入对气体水合物颗粒 间黏附力的影响，本文分别对质量分数为 $3.5 \mathrm{wt} . \%$ 和 $5 \mathrm{wt} . \%$ 的 $\mathrm{NaCl}$ 溶液液滴生成的水合物颗粒间黏附力进 行了测量. $3.5 \mathrm{wt} . \% \mathrm{NaCl}$ 共进行了 5 次独立重复实验, 测得的颗粒间作用力平均值为 $(16.3 \pm 1.2) \mathrm{mN} / \mathrm{m}$, 低于 $1 \mathrm{wt} . \% \mathrm{NaCl}$ 的颗粒间作用力和基准值. 这一结果说明 当 $\mathrm{NaCl}$ 浓度在 $1 \mathrm{wt} . \%$ 基础上继续升高时，水合物颗粒 间的作用力进一步下降. 然而, 当液滴中 $\mathrm{NaCl}$ 浓度继 续升高到 $5 \mathrm{wt} . \%$ 时，水合物颗粒间作用力并没有继续 下降，而是十分接近 $\mathrm{NaCl}$ 浓度为 $3.5 \mathrm{wt}$.\%时的数值， 4 组重复实验测量平均值为 $(16.3 \pm 1.7) \mathrm{mN} / \mathrm{m}$.

四组实验中水合物颗粒间作用力与 $\mathrm{NaCl}$ 的关系 如图 5 所示. 图中可见当 $\mathrm{NaCl}$ 质量分数低于 $3.5 \mathrm{wt} . \%$ 时, 水合物颗粒间作用力随 $\mathrm{NaCl}$ 浓度的升高而降低，但当 $\mathrm{NaCl}$ 质量分数达到 $5 \mathrm{wt} . \%$ 时，水合物颗粒间作用力与 $3.5 \mathrm{wt} . \%$ 时的作用力十分接近，并没有继续降低. 这一 实验结果表明, $\mathrm{NaCl}$ 的存在可以使水合物颗粒间的作 用力显著降低(作用力可降低至不含 $\mathrm{NaCl}$ 条件下的 $50 \%$ 以下)，但当 $\mathrm{NaCl}$ 的质量分数高于特定范围时(本 实验中为 $3.5 \mathrm{wt} \%), \mathrm{NaCl}$ 减小颗粒间作用力的效果不 会出现明显变化. Pagezy等人 ${ }^{[19]}$ 的研究也发现，对于 含 $\mathrm{NaCl}$ 的水合物浆，当 $\mathrm{NaCl}$ 浓度介于 $3 \%-10 \%$ 之间时，

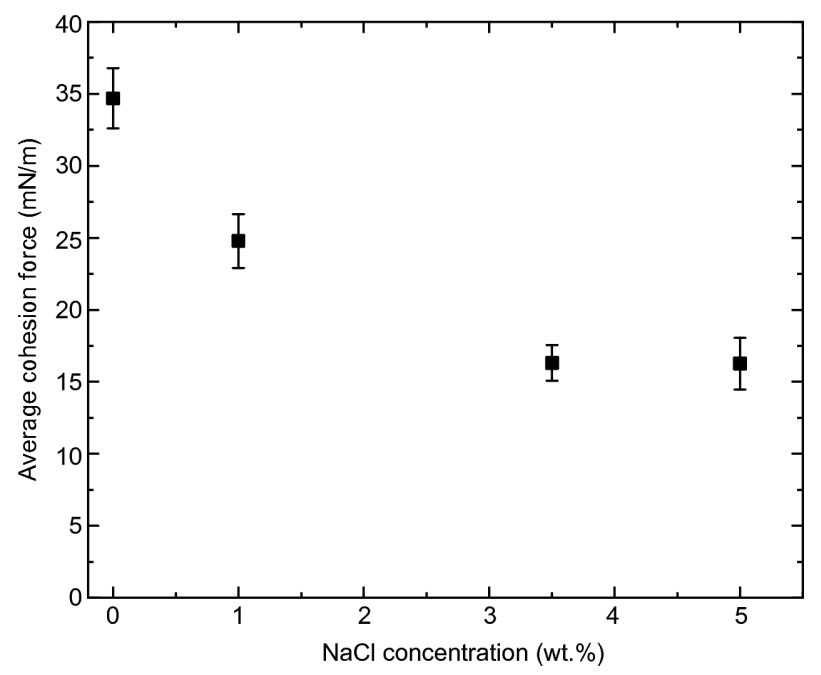

图 5 不同 $\mathrm{NaCl}$ 质量分数条件下水合物颗粒间作用力对比 Figure 5 Average force comparison of different $\mathrm{NaCl}$ concentrations (at least four repeats for the result of each $\mathrm{NaCl}$ concentration).
水合物浆的流动性会得到明显提升. 根据本文的实验 结果可以推测出这一现象的可能原因是加入 $\mathrm{NaCl}$ 后 水合物颗粒间黏附力降低，从而使颗粒聚集变得相对 困难.

由于目前并没有关于盐度对气体水合物颗粒间作 用力影响机理的完整研究, 因此, 在取得不同浓度 $\mathrm{NaCl}$ 条件下水合物颗粒间作用力后，为了解释盐度和 气体水合物颗粒间黏附力的关系，本文利用毛细液桥 理论模型对含 $\mathrm{NaCl}$ 条件下的水合物颗粒间作用力进 行了估算. 该模型假设当两个水合物颗粒发生接触时, 其各自表面的准液层会在颗粒间形成微小的毛细液桥 (Capillary Bridge), 水合物颗粒间的黏附力来自于两个 颗粒表面间隙中微量液体产生的毛细管力 ${ }^{[14,20]}$. 其基 本方程如下:

$$
\frac{F_{\mathrm{a}}}{R^{*}}=2 \pi \gamma \sin \alpha \sin \left(\theta_{\mathrm{p}}+\alpha\right)+\frac{2 \pi \gamma \cos \theta_{\mathrm{p}}}{1+\frac{H}{2 d_{s p / s p(H, V)}}},
$$

式中, $F_{\mathrm{a}}$ 为颗粒间作用力, $\gamma$ 为液体在颗粒界面处的表 面张力, $\theta_{\mathrm{p}}$ 为液体与水合物颗粒表面的接触角, $R^{*}$ 是等 效半径, 为两个颗粒半径的调和平均数, 由式(4)计算. 其他参数如图6所示. 式(3)中的浸没深度 $d_{s p / s p(H, V)}$ 和毛 细液桥体积 $V$ 分别由式(5)和(6)计算:

$$
\begin{aligned}
& R^{*}=\frac{2 R_{1} R_{2}}{R_{1}+R_{2}}, \\
& d_{s p / s p(H, V)}=\left(\frac{H}{2}\right) \times\left[-1+\sqrt{1+\frac{2 V}{\pi R H^{2}}}\right],
\end{aligned}
$$

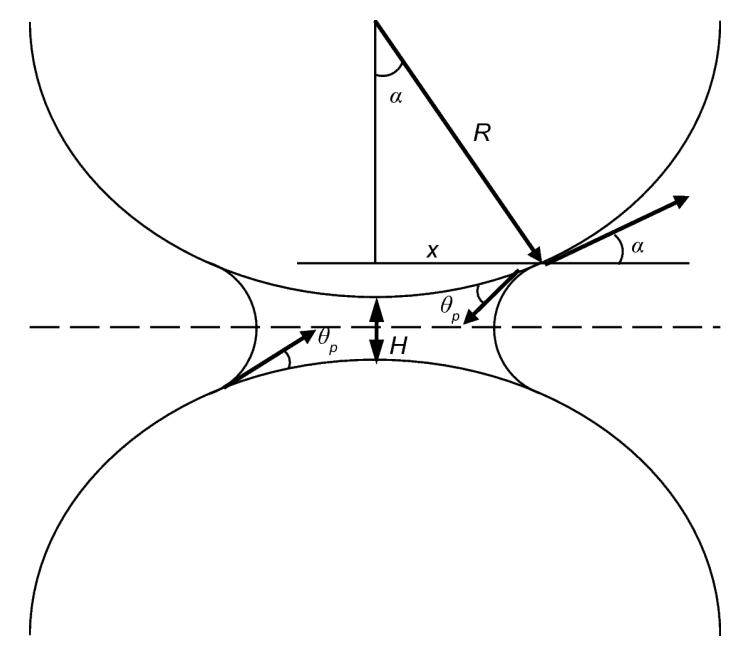

图 6 颗粒-颗粒间毛细液桥作用示意图

Figure 6 Geometry of the sphere/sphere interaction with a capillary liquid bridge. 
$V=\pi R^{2} \alpha^{2} H+0.5 \pi R^{3} \alpha^{4}$.

根据式(3), (5)和(6)可知, 要计算 $F_{\mathrm{a}}$, 首先需要获得 以下几个参数: (1) 两个颗粒的等效半径 $R^{*} ;(2)$ 不同浓 度的 $\mathrm{NaCl}$ 溶液在水合物颗粒表面的液-固接触角 $\theta_{\mathrm{p}}$ 、 表面张力 $\gamma$ 和拥抱角 $\alpha$; (3) 接触时颗粒间的最小间距 $H$.

其中等效半径 $R{ }^{*}$ 通过测量两个颗粒的半径并由 式(4)计算得出, 拥抱角 $\alpha$ 和最小间距 $H$ 采用文献中相似 实验条件下的数值, 分别为 $0.1^{\circ}$ 和 $50 \mathrm{~nm}^{[14]}$. 此外, 通过 查阅文献可知, 实验温度下 $\mathrm{NaCl}$ 溶液的表面张力随盐 度的增大而上升，两者间的对应关系如图7(a)所示 ${ }^{[21]}$. 目前并没有 $\mathrm{NaCl}$ 溶液在气体水合物表面接触角的相 关数据，但Hirata等人 ${ }^{[22]}$ 的研究表明水合物表面具有 亲水性，并且Sghair等人 ${ }^{[23]}$ 发现 $\mathrm{NaCl}$ 溶液在亲水表面 的接触角随着 $\mathrm{NaCl}$ 的浓度升高而逐渐增大，其实验结 果如图7(b)所示.

根据上述参数，利用毛细液桥模型对 $0 \mathrm{wt} . \%-$ 5 wt. \% NaCl液滴生成的水合物颗粒间黏附力进行了
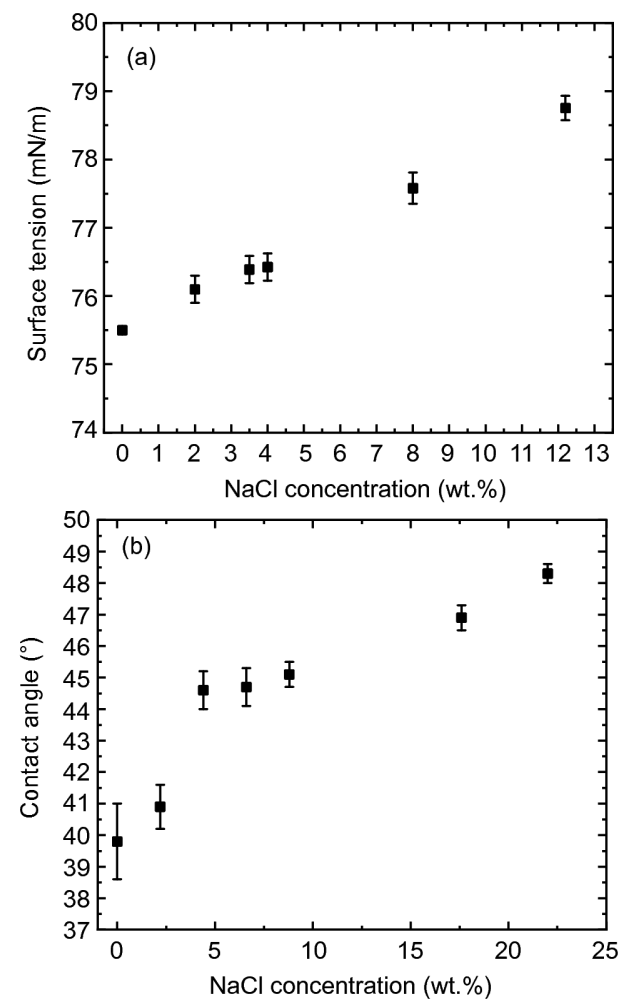

图 7 水的表面张力及亲水表面接触角随 $\mathrm{NaCl}$ 浓度变 化 ${ }^{[21,23]}$. (a) 表面张力; (b) 接触角

Figure 7 (a) Effects of salinity on water surface tension. (b) Evolution of contact angle as a function of $\mathrm{NaCl}$ concentration for the hydrophilic glass samples [21,23].
估算, 计算结果如图8所示. 从图中可以发现, 通过毛 细液桥理论模型计算得出的水合物颗粒间作用力同样 随着 $\mathrm{NaCl}$ 浓度的升高而降低. 这一结论表明, 接触角 的变化是水合物颗粒间作用力下降的一个可能因素.

\section{2 碳钢表面腐蚀程度对黏附力的影响}

在水合物开采过程中，管道壁面长期处于潮湿含 盐的环境下, 表面极易出现电化学腐蚀. 因此, 为了研 究管道壁面腐蚀程度对水合物颗粒-壁面间黏附力的 影响, 采用碳钢片模拟管道表面, 并在实验前将其浸泡 在 $\mathrm{NaCl}$ 溶液中, 以模拟实际开采过程中管道表面的腐 蚀情况. 碳钢片的不同表面腐蚀程度由在 $5 \mathrm{wt} . \%$ 的 $\mathrm{NaCl}$ 溶液中浸泡的时间长短决定. 图9所示为碳钢片 样品在 $\mathrm{NaCl}$ 溶液中分别浸泡 $0,24,48,72,96$ 和 $120 \mathrm{~h}$ 后的照片. 图中可见, 浸泡时间较短时 $(<24 \mathrm{~h})$, 碳钢片 表面仍存在少量未被腐蚀区域, 随着碳钢片在 $\mathrm{NaCl}$ 溶 液中浸泡时间的延长, 锈迹逐渐开始覆盖碳钢片样品 的整个表面.

在完成上述准备工作后，对于每个碳钢片样品与 $\mathrm{CH}_{4}-\mathrm{C}_{2} \mathrm{H}_{6}$ 水合物颗粒间的黏附力进行了 3 次重复实验, 图10中所示为测量结果中 4 个典型阶段的黏附力分布. 对于无腐蚀的碳钢片，水合物颗粒与其表面接触力的 频率分布近似正态分布，由于实验过程中水合物颗粒 与界面的接触点是随机选取的, 因此这一分布形态说

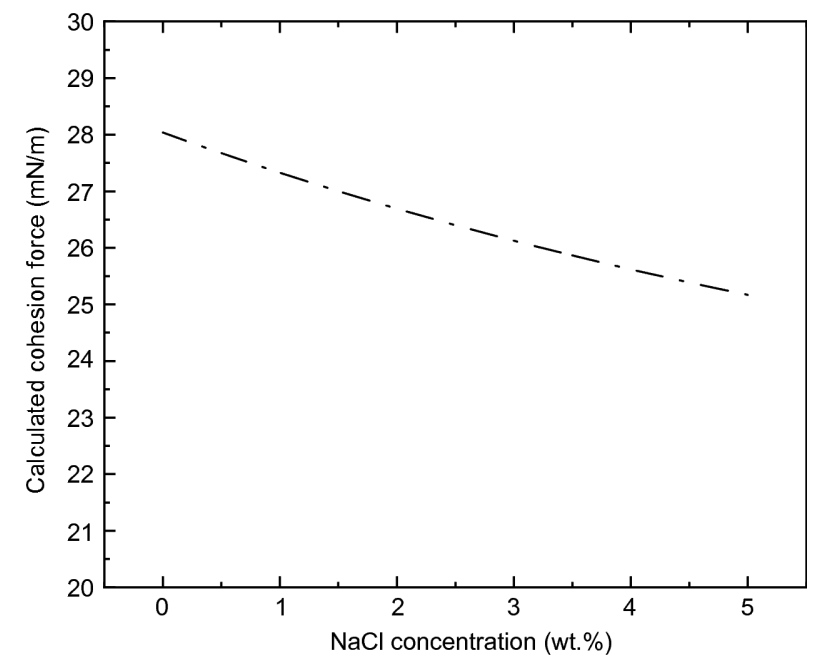

图 8 毛细液桥模型计算得出的水合物颗粒间作用力与盐 度的关系

Figure 8 Cohesion force between hydrate particles calculated by capillary bridge model as a function of salinity. 


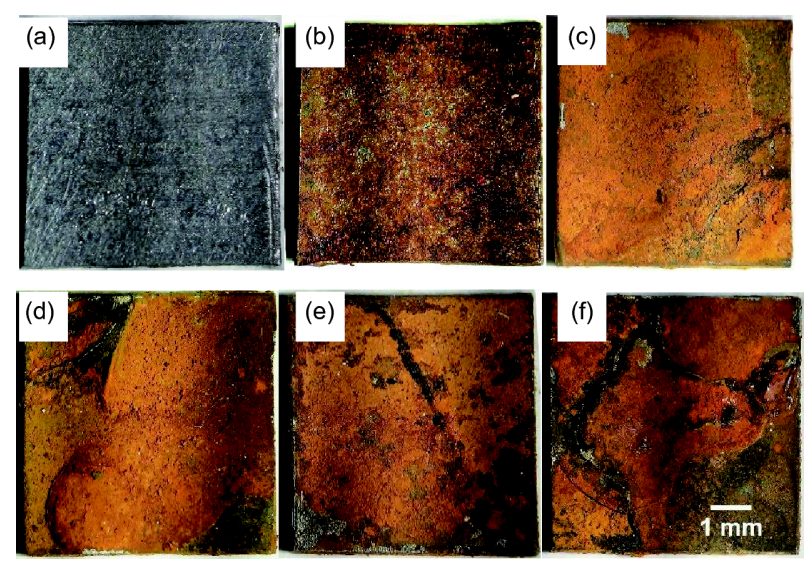

图 9 (网络版彩图)在 $5 \mathrm{wt} . \% \mathrm{NaCl}$ 溶液中浸泡不同时长后的 碳钢片. (a) 0 h, (b) 24 h, (c) 48 h, (d) 72 h, (e) 96 h, (f) 120 h Figure 9 (Color online) Surface conditions of CS samples with different soak times in 5 wt.\% NaCl solution. (a) Pristine; (b) $24 \mathrm{~h}$; (c) $48 \mathrm{~h}$; (d) $72 \mathrm{~h}$; (e) $96 \mathrm{~h}$; (f) $120 \mathrm{~h}$.

明碳钢片各处的表面特性基本一致(图10(a))。当碳钢 片在 $\mathrm{NaCl}$ 溶液中浸泡 $24 \mathrm{~h}$ 后，水合物颗粒与其之间的 作用力分布如图10(b)所示. 作用力的频率分布由近似 正态分布转变为双峰形分布, 出现这一分布形态的原 因是由于此时碳钢片表面只是部分腐蚀，依然存在许 多未被腐蚀的区域(如图9(b)所示), 因此在这两类区域 上的颗粒-表面接触时的作用力并不相同, 最终呈现出 双峰式的频率分布. 图10(c)所示为碳钢片在 $\mathrm{NaCl}$ 溶液 中浸泡 $48 \mathrm{~h}$ 后与水合物颗粒间作用力的频率分布. 可 以发现分布形式又回归到近似正态分布，这是由于碳 钢片表面在经过 $48 \mathrm{~h}$ 的浸泡后已经基本被 $\mathrm{FeOOH}, \mathrm{Fe}_{2}$ $\mathrm{O}_{3}$ 和 $\mathrm{Fe}_{3} \mathrm{O}_{4}$ 等腐蚀过程的产物覆盖(如图9(c)), 因此表 面各处的水合物膜厚度和形态都比较接近. 图10(d)所 示为碳钢片在 $\mathrm{NaCl}$ 溶液中浸泡 $120 \mathrm{~h}$ 后的作用力频率 分布, 其分布形式介于正态分布与双峰分布之间, 这是 由于经过 $\mathrm{NaCl}$ 溶液 $120 \mathrm{~h}$ 的浸泡后, 碳钢片表面虽然已 经完全被腐蚀产物覆盖, 但局部出现了腐蚀产物堆积/ 生长的不均匀(图9(f)), 从而使得表面各处与水合物颗 粒间的作用力出现分化. 但由于整体已经被腐蚀产物
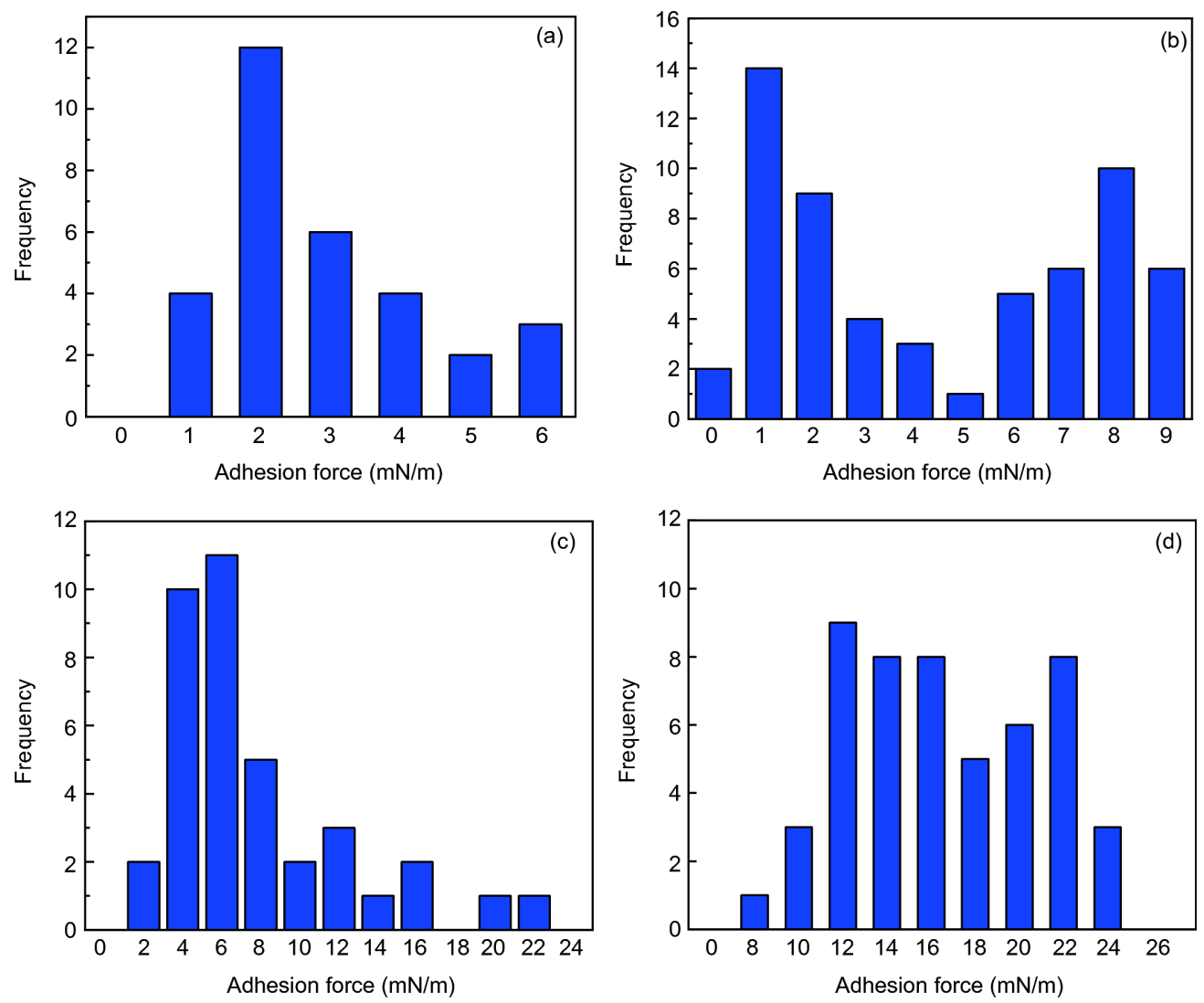

图 10 (网络版彩图)不同腐蚀程度碳钢片与水合物颗粒间作用力分布. (a) 无腐蚀; (b) 浸泡 $24 \mathrm{~h} ;$ (c) 浸泡 $48 \mathrm{~h}$; (d) 浸泡 $120 \mathrm{~h}$ Figure 10 (Color online) Typical adhesion force distributions. (a) Pristine; (b) $24 \mathrm{~h}$ soak; (c) $48 \mathrm{~h}$ soak; (d) $120 \mathrm{~h}$ soak. 
覆盖, 所以各处的作用力大小差距并不显著, 最终呈现 出如图所示的分布.

碳钢片与水合物颗粒间作用力随表面腐蚀程度的 变化如图11所示. 图中各数据点均由 3 次以上重复实验 取平均值并计算误差限得出. 由图中数据可以看出, 水 合物颗粒与碳钢片表面间的作用力随着碳钢片在 $\mathrm{NaCl}$ 溶液中浸泡时间的延长而增大，这表明碳钢的表 面腐蚀对其表面自由水/水合物膜的形成和分布有显 著影响. 当碳钢片的浸泡时间达到 $96 \mathrm{~h}$ 后，此时的颗 粒-表面作用力平均值与 $120 \mathrm{~h}$ 的作用力相比几乎没有 差别，但 $120 \mathrm{~h}$ 的测量值误差显著增大. 导致这一结果 的可能原因是此时碳钢片表面已经完全被腐蚀产物覆 盖, 腐蚀速率显著降低, 但表面局部却可能因为浸泡时

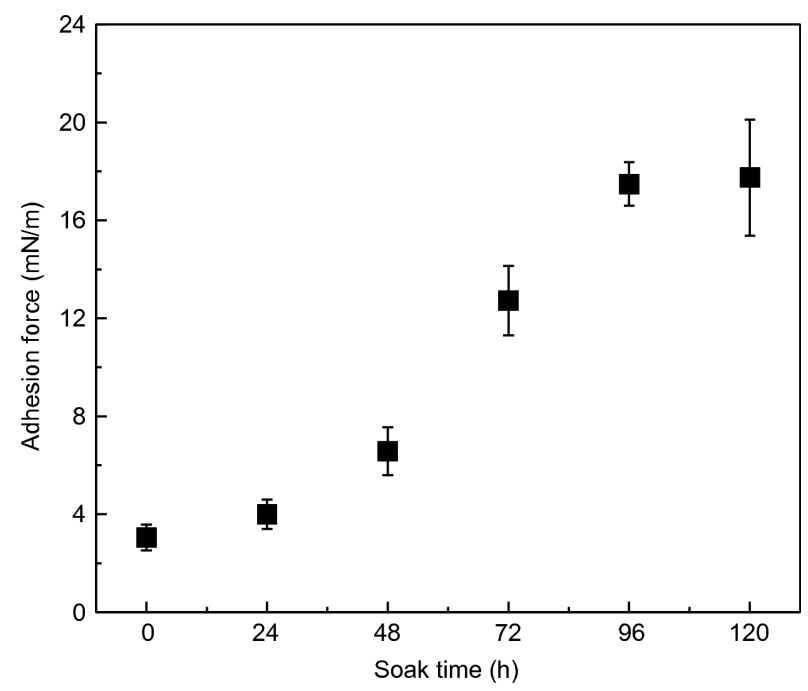

图 11 水合物颗粒与不同腐蚀程度的碳钢片表面间作用力 Figure 11 Adhesion force comparison of $\mathrm{CH}_{4} / \mathrm{C}_{2} \mathrm{H}_{6}$ hydrate particle - CS samples with different extents of corrosion (at least three repeats for each data point).
间的延长出现腐蚀产物分布的不均匀，并因此导致测 量过程中各处的作用力差别较大.

对比未腐蚀碳钢表面和在 $\mathrm{NaCl}$ 溶液中浸泡 $120 \mathrm{~h}$ 后的碳钢表面与水合物颗粒间的作用力可以发现, 由 于表面腐蚀的存在，颗粒的表面附着力增加了 $500 \%$ 左 右. 这一结果表明管道壁面的表面处理(疏水、防腐 等)对于降低水合物资源开采和油气输运过程中水合 物颗粒的聚集和沉积风险具有重要意义.

\section{4 结论}

本文研究了 $\mathrm{NaCl}$ 溶液浓度及腐蚀对于 $\mathrm{CH}_{4} / \mathrm{C}_{2} \mathrm{H}_{6}$ 混合气生成的II型水合物颗粒黏附力的影响. 利用气 体水合物颗粒黏附力测量系统, 首次实现了高压下 $\mathrm{CH}_{4} / \mathrm{C}_{2} \mathrm{H}_{6}$ 生成的II型水合物颗粒间、颗粒-碳钢表面间 黏附力的原位测量. 首先研究了不同浓度的 $\mathrm{NaCl}$ 溶液 液滴生成的气体水合物颗粒间黏附力变化规律, 发现 $\mathrm{NaCl}$ 的存在会显著降低气体水合物颗粒间的黏附力, 而当 $\mathrm{NaCl}$ 浓度高于 $5 \mathrm{wt} . \%$ 时，颗粒间黏附力不会随 $\mathrm{NaCl}$ 浓度的升高而进一步下降. 为了解释 $\mathrm{NaCl}$ 对水合 物颗粒黏附力的影响机制, 利用毛细液桥理论模型对 含盐条件下水合物颗粒间黏附力进行了估算, 其结果 表明颗粒间黏附力降低的可能原因是 $\mathrm{NaCl}$ 的存在增 大了毛细液桥与水合物颗粒表面的接触角. 其次, 通过 将碳钢片样品在 $\mathrm{NaCl}$ 溶液中浸泡模拟管壁在海水条 件下的腐蚀, 研究了水合物颗粒与腐蚀存在条件下的 碳钢表面间黏附力, 发现由于 $\mathrm{NaCl}$ 溶液导致的碳钢表 面腐蚀能够显著提高水合物颗粒与碳钢表面的黏附 力, 最高可以达到无腐蚀条件下的 $500 \%$. 上述结论有 助于进一步理解水合物开采和油气输运过程中深水管 道内的气体水合物颗粒聚集和沉积机制.

\section{参考文献}

1 Sloan E D, Koh C A. Clathrate Hydrates of Natural Gases. Boca Raton: CRC Press, 2008

2 Sloan E D, Koh C A, Sum A. Natural Gas Hydrates in Flow Assurance. Houston: Gulf Professional Publishing, 2010

3 Rao I, Koh C A, Sloan E D, et al. Gas hydrate deposition on a cold surface in water-saturated gas systems. Ind Eng Chem Res, 2013, 52: 62626269

4 Di Lorenzo M, Aman Z M, Kozielski K, et al. Underinhibited hydrate formation and transport investigated using a single-pass gas-dominant flowloop. Energy Fuels, 2014, 28: 7274-7284

5 Di Lorenzo M, Aman Z M, Sanchez Soto G, et al. Hydrate formation in gas-dominant systems using a single-pass flowloop. Energy Fuels, 2014, 28: $3043-3052$ 
6 Turner D J. Clathrate hydrate formation in water-in-oil dispersions. Dissertation for Doctoral Degree. Golden: Colorado School of Mines, 2006

7 Yang S, Kleehammer D M, Huo Z, et al. Temperature dependence of particle-particle adherence forces in ice and clathrate hydrates. J Colloid Interface Sci, 2004, 277: 335-341

8 Taylor C J, Dieker L E, Miller K T, et al. Micromechanical adhesion force measurements between tetrahydrofuran hydrate particles. J Colloid Interface Sci, 2007, 306: 255-261

9 Dieker L E, Aman Z M, George N C, et al. Micromechanical adhesion force measurements between hydrate particles in hydrocarbon oils and their modifications. Energy Fuels, 2009, 23: 5966-5971

10 Brown E, Khan M N, Salmin D, et al. Cyclopentane hydrate cohesion measurements and phase equilibrium predictions. J Nat Gas Sci Eng, 2016, 35: $1435-1440$

11 Lee B R, Koh C A, Sum A K. Mechanism of cohesive forces of cyclopentane hydrates with and without thermodynamic inhibitors. Ind Eng Chem Res, 2014, 53: 18189-18193

12 Aspenes G, Dieker L E, Aman Z M, et al. Adhesion force between cyclopentane hydrates and solid surface materials. J Colloid Interface Sci, 2010, 343: 529-536

13 Smith J D, Meuler A J, Bralower H L, et al. Hydrate-phobic surfaces: Fundamental studies in clathrate hydrate adhesion reduction. Phys Chem Chem Phys, 2012, 14: 6013-6020

14 Aman Z M, Brown E P, Sloan E D, et al. Interfacial mechanisms governing cyclopentane clathrate hydrate adhesion/cohesion. Phys Chem Chem Phys, 2011, 13: 19796-19806

15 Aman Z M, Leith W J, Grasso G A, et al. Adhesion force between cyclopentane hydrate and mineral surfaces. Langmuir, 2013, 29: 15551-15557

16 Lee B R, Sum A K. Micromechanical cohesion force between gas hydrate particles measured under high pressure and low temperature conditions. Langmuir, 2015, 31: 3884-3888

17 Rabinovich Y I, Esayanur M S, Moudgil B M. Capillary forces between two spheres with a fixed volume liquid bridge: Theory and experiment. Langmuir, 2005, 21: 10992-10997

18 Servio P, Englezos P. Morphology of methane and carbon dioxide hydrates formed from water droplets. AIChE J, 2003, 49: 269-276

19 Pagezy L, Glenat P, Bourg P, et al. Good transportability of liquid hydrates slurries made from just salty waters (from 3 to $10 \%$ wt. NaCl) and gas. In: 9th International Conference on Gas Hydrates, 2017. 2183

20 Zhao Y P. Nano and Mesoscopic Mechanics (in Chinese). Beijing: Science Press, 2014 [赵亚溥. 纳米与介观力学. 北京: 科学出版社, 2014]

21 Nayar K G, Panchanathan D, McKinley G H, et al. Surface tension of seawater. J Phys Chem Ref Data, 2014, 43: 043103

22 Hirata A, Mori Y H. How liquids wet clathrate hydrates. Chem Eng Sci, 1998, 53: 2641-2643

23 Sghaier N, Prat M, Ben Nasrallah S. On the influence of sodium chloride concentration on equilibrium contact angle. Chem Eng J, 2006, 122: $47-53$ 


\title{
The effect of $\mathrm{NaCl}$ concentration and corrosion on cohesion/ adhesion forces of structure-II hydrate particles
}

\author{
WANG ShengLong ${ }^{1}$, Fan ShuanShi ${ }^{*}$, SONG YongChen ${ }^{2}$, LANG XueMei ${ }^{1}$, \\ WANG YanHong ${ }^{1}$, LI Gang ${ }^{1} \&$ CHEN JianBiao ${ }^{1}$ \\ ${ }^{1}$ Key Laboratory of Heat Transfer Enhancement and Energy Conservation of Education Ministry, School of Chemistry and Chemical \\ Engineering, South China University of Technology, Guangzhou 510640, China; \\ ${ }^{2}$ Key Laboratory of Ocean Energy Utilization and Energy Conservation of Education Ministry, School of Energy and Power \\ Engineering, Dalian University of Technology, Dalian 116024, China
}

Gas hydrates are ice-like solid compounds formed from water and suitably sized gas molecules under high pressure and low temperature conditions. In typical oil and gas transportation flowlines, when water is present gas hydrates particles can form, agglomerate, and accumulate in the flowlines. These particles can also deposit on flowline walls during fluid flow or during shut-in/restart conditions. The formation of hydrate plugs can cause a blockage in the flowline, as well as other safety concerns. Although researchers have studied how temperature and annealing time would affect the cohesion/ adhesion forces between hydrate particles and particle-surface systems, the effects of surface corrosion, salinity and water amount on the adhesion force have not been reported. Deep-water flowlines typically contain brine, and water condensation and corrosion can occur on flowline walls. A water layer and corrosion on flowline walls can affect hydrate film growth and deposition, and salt dissolved in the liquid phase may also influence the agglomeration of hydrate particles. Thus, the effects of free water, corrosion and salt on $\mathrm{CH}_{4} / \mathrm{C}_{2} \mathrm{H}_{6}$ hydrate particle-particle and particle-surface micromechanical forces are essential for advancing the understanding on hydrate agglomeration and deposition mechanisms in gas dominated deep-water pipelines. In order to investigate the mechanism of gas hydrate deposition and agglomeration in gas dominated flowlines, a high-pressure micromechanical force (MMF) apparatus was applied to directly measure $\mathrm{CH}_{4} / \mathrm{C}_{2} \mathrm{H}_{6}$ hydrate adhesion/cohesion forces. The influence of $\mathrm{NaCl}$ concentration on the cohesion force between $\mathrm{CH}_{4} / \mathrm{C}_{2} \mathrm{H}_{6}$ hydrate particles was studied, and the effect of surface corrosion on adhesion forces between hydrate particles and carbon steel (CS) were analysed. It was found that the presence of $\mathrm{NaCl}$ decreased the cohesion force between hydrate particles, and a possible explanation of this phenomenon was given based on the capillary liquid bridge model. For the adhesion force measurements, the results indicated that with increasing visual corrosion, the adhesion force between gas hydrate particle and CS surface increased by up to $500 \%$.

gas hydrates, cohesion force, salinity, corrosion, flow assurance

PACS: 89.30.an, 89.30.aj, 61.46.Df, 81.70.Fy, 51.10.+y

doi: $10.1360 /$ SSPMA2018-00169 